\title{
Mesospheric radar wind comparisons at high and middle southern latitudes
}

\author{
lain M. Reid ${ }^{1,2^{*}} \mathbb{0}$, Daniel L. McIntosh² ${ }^{2}$ Damian J. Murphy ${ }^{3}$ and Robert A. Vincent ${ }^{2}$
}

\begin{abstract}
We compare hourly averaged neutral winds derived from two meteor radars operating at 33.2 and $55 \mathrm{MHz}$ to estimate the errors in these measurements. We then compare the meteor radar winds with those from a mediumfrequency partial reflection radar operating at $1.94 \mathrm{MHz}$. These three radars are located at Davis Station, Antarctica. We then consider a middle-latitude $55 \mathrm{MHz}$ meteor radar wind comparison with a $1.98 \mathrm{MHz}$ medium-frequency partial reflection radar to determine how representative the Davis results are. At both sites, the medium-frequency radar winds are clearly underestimated, and the underestimation increases from $80 \mathrm{~km}$ to the maximum height of $98 \mathrm{~km}$. Correction factors are suggested for these results.
\end{abstract}

Keywords: Medium-frequency partial reflection radar, Meteor radar, Davis station, Winds

\section{Introduction}

One of the oldest radar techniques for investigating the mesosphere lower thermosphere (MLT) region between 60 and $100 \mathrm{~km}$ uses medium frequencies (MF) and partial reflections (PR) from that height region to measure electron densities using the differential absorption experiment (DAE) (see, e.g., Holdsworth et al. 2002), to measure neutral winds, most commonly using spaced antennas (SA) for reception, together with the full correlation analysis (FCA) (see, e.g., Briggs 1984), to measure turbulent strength using velocity variances (see, e.g., Holdsworth et al. 2001), and to measure structure using variations in returned power with height (see, e.g., Reid 1990). The FCA is arguably no longer the best analysis available for use on these radars, but there is a large archival data set, and many MF PR radars are still using it. Given the longevity of its use, it is not surprising that there have been numerous intercomparisons with other techniques. These began in the 1960s and have continued until the present.

The intercomparison of different techniques for measuring the same parameters serves three main purposes:

*Correspondence: ireid@atrad.com.au

${ }^{1}$ ATRAD Pty Ltd, Thebarton, Australia

Full list of author information is available at the end of the article
1. Validation

2. Calibration, and

3. Better understanding the relative strengths and weaknesses of each technique.

A review of the MF PR technique, a discussion of its strengths and weaknesses, and a summary of previous intercomparisons with other techniques is provided by Reid (2015). The interested reader is directed there for an in-depth discussion. Here, we can briefly summarize attempts at validation of MF PR radars using SA and the FCA in some general statements. These are that in the 80-94 km height region, MF PR SA radars using the FCA and DAE analyses generally:

(1) Underestimate the wind magnitudes, with the underestimation being height dependent, increasing from 80 to $94 \mathrm{~km}$, and are dependent on the particular radar hardware and software implementation.

(2) Correctly measure the wind direction.

(3) Provide a measure of the electron density.

(4) Provide an upper limit to the turbulent intensity. In addition:

(5) Above about 92-94 $\mathrm{km}$ at midlatitudes, receiver saturation effects due to E-region total reflections 
may contaminate the radar returns (see, e.g., Hocking (1997)), particularly with wide beam MF PR radars (such as the Davis MF radar), with both the magnitude and direction being potentially affected, and these results should be avoided unless additional information on the total reflection height is available.

(6) In the $60-80 \mathrm{~km}$ height region, there are too few intercomparisons to reach a general statistical conclusion on the validity of the winds.

Given this general understanding, more recent intercomparisons have been for the purposes of calibrating MF PR results against meteor radars (e.g., Wilhelm et al. 2017). Cervera and Reid (1996) did do this somewhat earlier, but only for the zonal wind component. In another earlier MF PR radar calibration, Manson et al. (1992) compared MF PR radar FCA winds, EISCAT and SOUSY VHF radar winds and rocket falling sphere and chaffderived winds at Troms $\varnothing$ and found in the $84-90.5 \mathrm{~km}$ height region that the amplitude ratio of the MF PR FCA winds had a median value of 0.63 and a mean value of 0.60 when compared to the other techniques. These values were then used to recalibrate the MF PR winds. In addition, we note in this context that Dolman and Reid (2014) successfully calibrated VHF SA FCA winds for operational wind profilers in the boundary layer and troposphere using radiosonde winds as the calibration reference. Meteor radars have become relatively common, and the de facto reference for wind measurements in the $80-100 \mathrm{~km}$ height region, although naturally they suffer from their own limitations (see, e.g., Reid 2015), and most recent MF PR radar wind comparisons are with meteor radar winds.

It is also useful to compare similar radar systems to determine uncertainties in the parameters they produce. This may be by intercomparison of different radar channels from the same radar being used to measure the same target region (e.g., Reid and Vincent 1987), collocated radars of the same type, but different frequencies, observing the same targets (e.g., Younger et al. 2008), or intercomparison of the same radar types being operated in different analysis modes on the same data (Reid 1988; Holdsworth et al. 1996; Holdsworth and Reid 2004a, b).

In this paper, we compare winds from two collocated meteor radars at Davis Station Antarctica, determine the errors inherent in these winds from this comparison, and then use these to compare the meteor radar winds with winds from a collocated MF PR radar. We then consider a similar comparison made at the Buckland Park (BP) field site near Adelaide, Australia.

\section{Equipment}

\section{Davis station}

The two meteor radar systems at Davis Station are a dedicated 33.2 MHz 'all-sky' interferometric meteor radar from the ATRAD series, essentially identical to that described by Holdsworth et al. (2004a, b), and a $55 \mathrm{MHz}$ 'all-sky' interferometric meteor radar 'piggy-backed' onto an stratosphere-troposphere (ST) radar (Holdsworth et al. 2006) which is also an ATRAD series radar. The operating parameters for the $33.2 \mathrm{MHz}$ system are summarized in Table 1. Operational modes and results from the meteor mode of the $55 \mathrm{MHz}$ system have previously been described by Holdsworth et al. (2006), and Reid et al. (2006), but briefly, this radar operates continuously with a sequence of experiments comprising of ST, meteor, and in summer, Polar Mesospheric Summer Echo (PMSE) experiments. This reduces the observation time in meteor mode and hence the meteor count rates. The peak transmitted powers and duty cycles are also different, being $7.5 \mathrm{~kW}$ at $4 \%$ for the $33.2 \mathrm{MHz}$ radar and $18 \mathrm{~kW}$ at $3 \%$ for the $55 \mathrm{MHz}$ radar.

There is a dependence of count rate $N$ on both transmitted power and frequency, given by

$$
N \propto \frac{P_{\mathrm{T}}^{1 / 2} G \lambda^{3 / 2}}{P_{\mathrm{R}}^{1 / 2}}
$$

where $P_{\mathrm{T}}$ is the transmitted power, $P_{\mathrm{R}}$ is the received power, $G$ is the system gain, and $\lambda$ is the transmitted wavelength (McKinley 1961). This relationship was validated by McIntosh (2010) for the Buckland Park $55 \mathrm{MHz}$ ST/meteor radar, which is essentially identical to the Davis $55 \mathrm{MHz}$ radar.

These two variations between the radars and their operating modes combine to produce differences in the meteor count rates between them. For example, for all of 2006 , the mean daily count was around 9600 , with a peak mean daily echo rate of about 1700 meteor detections near $90 \mathrm{~km}$ for the $33 \mathrm{MHz}$ radar, and a daily mean count of about 2100 , with a peak mean daily count of about 390 meteor detections near $88 \mathrm{~km}$ for the $55 \mathrm{MHz}$ radar, noting also that the meteor peak detection height decreases with increasing frequency. The height time and height distributions for the two meteor radars and the mean profiles for 2006 are shown in Fig. 1. Further discussion on Davis meteor radar count rates and their daily and seasonal variations may be found in Reid et al. (2006) and Holdsworth et al. (2008).

For the observations presented here, both meteor radars used a pair of two-element gamma-matched crossed dipole antennas for transmission, with reception 
Table 1 Typical radar parameter sets used on the Davis and BP meteor and MF PR radars for the work described here

\begin{tabular}{|c|c|c|c|c|c|c|}
\hline Parameter & Davis 33.2 & Davis 55 & BP 55 & Davis 1.94 & BP 1.98 & Units \\
\hline Carrier frequency & 33.2 & 55 & 55 & 1.94 & 1.98 & $\mathrm{MHz}$ \\
\hline Carrier wavelength & 9.04 & 5.45 & 5.45 & 154.6 & 151.5 & $\mathrm{~m}$ \\
\hline Pulse width & 3600 & 2275 & 7200 & 3200 & 4000 & $\mathrm{~m}$ \\
\hline $\begin{array}{l}\text { Pulse repetition } \\
\text { frequency }\end{array}$ & 430 & 1960 & 430 & 100 & 100 day/20 night & $\mathrm{Hz}$ \\
\hline Pulse type & Gaussian modulated & Gaussian modulated & Gaussian modulated & Gaussian modulated & Gaussian modulated & \\
\hline Range resolution & 2 & 2 & 2 & 2 & 2 & $\mathrm{~km}$ \\
\hline Range aliasing & No & Yes & $\mathrm{No}$ & No & No & \\
\hline Sampling range start & 70 & 75 & 70 & 40 & 50 & $\mathrm{~km}$ \\
\hline Sampling range finish & 314.8 & 300 & 314.8 & 108 & 158 & $\mathrm{~km}$ \\
\hline Peak power & 7.5 & $\begin{array}{l}18 \text { (one of six } 18 \mathrm{~kW} \\
\text { tube transmitter } \\
\text { modules) }\end{array}$ & 18 and $83 \mathrm{~kW}$ & 32 & 92 & $\mathrm{~kW}$ \\
\hline Coding & 4-bit Complementary & None & None & None & None & \\
\hline Duty cycle & 4.13 & 2.97 & 2.97 & 0.2 & 0.2 & $\%$ \\
\hline Polarization & Linear & Linear & Circular & $\mathrm{O} / \mathrm{X}$ & $\mathrm{O} / \mathrm{X}$ & \\
\hline
\end{tabular}

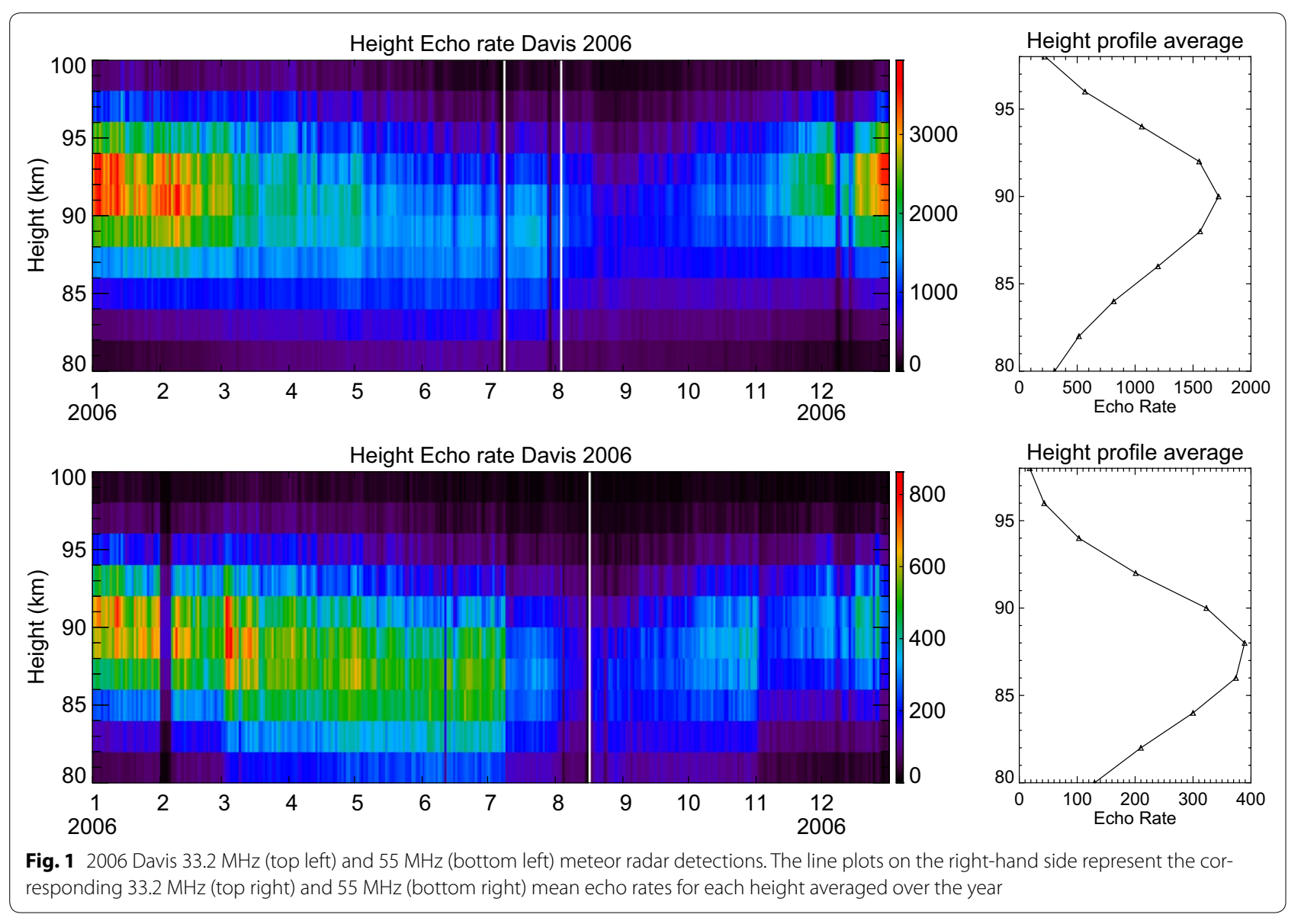


on five two-element folded dipoles arranged as an interferometer (see, e.g., Holdsworth et al. 2004a, b). Crossed two-element folded dipoles were added to the $33.2 \mathrm{MHz}$ radar for reception in the 2007/2008 austral summer season, but results from after this upgrade are not discussed here. Typical system parameters are summarized in Table 1. Additional discussion on the calibration of these radars for angle of arrival (AoA) and other phase calibration aspects may be found in Holdsworth et al. (2004a, b), Younger et al. (2013), and Younger and Reid (2017).

There is one other point to note about these meteor radar systems. The linear antennas are aligned $45^{\circ}$ from north in the NE and SW quadrants, and consequently, there is a low detection region associated with their endon view. It is expected that any effects due to this would equally affect winds in both the zonal and meridional directions.

The Davis Station MF PR radar began operation in 1994 after being moved from Mawson Station, Antarctica, and it was upgraded in 2004 to a higher transmitter power. It operates in spaced-antenna mode at a frequency of $1.94 \mathrm{MHz}$ and uses pulsed transmission with half-power full pulse widths of $30 \mu \mathrm{s}$, which corresponds to a range resolution of about $4 \mathrm{~km}$. Data are taken every $2 \mathrm{~min}$ and oversampled at $2 \mathrm{~km}$ height intervals. The raw data are analyzed using the FCA method to derive the horizontal winds (see, e.g., Briggs 1984). Other relevant system parameters are summarized in Table 1.

The Davis MF radar operates alternately in two separate modes of circular polarization transmission, $\mathrm{O}$-mode and $\mathrm{X}$-mode. The presence of Earth's magnetic field results in differences in the propagation characteristics of the two characteristic modes. The X-mode is affected more by absorption and group retardation in an ionized atmosphere in comparison with the O-mode (see, e.g., Tsutsumi and Aso 2005). Both modes of operation provide wind estimates over most of the height range depending upon time of year, and the results from both modes can be combined to create a measurement average (see, e.g., Holdsworth et al. 2001).

During the course of this study, it became apparent that the X-mode wind magnitudes on the Davis radar underestimated the $\mathrm{O}$-mode magnitudes, and further investigation was required. This work will be presented elsewhere. This difference was not evident at the Buckland Park field site. Consequently, we will only consider the Davis MF PR radar O-mode results here. The MF radar 2-min wind estimates were averaged to produce hourly averages to match the meteor radar hourly averaged estimates.

\section{Buckland Park}

There was one meteor radar at the Buckland Park field site during the time of the observations presented here, and this was essentially identical to the Davis Station $55 \mathrm{MHz} \mathrm{ST} /$ meteor hybrid radar. Two different transmitter configurations were used over the course of the observations presented here. The first used one (of six) $18 \mathrm{~kW}$ section an ATRAD VTX (tube) transmitter, as used at Davis Station. The second used the entire transmitter with a measured $83 \mathrm{~kW}$ of peak power. Transmission was on a single high-power crossed folded dipole single reflector antenna, with reception on five crossed gamma-matched two-element antennas arranged as an interferometer. The system parameters are summarized in Table 1.

The BP MF PR radar is described by Reid et al. (1995) and by Holdsworth and Reid (2004a, b), and the system parameters for this radar for the comparisons described here are given in Table 1.

\section{Results and discussion}

We start this section by briefly outlining the approach used to compare two data sets which represent measurements of the same quantity using two different systems and follow Hocking et al. (2001). The variance $\left(\eta_{x}^{2}\right)$ of a data set $\left\{x_{i}\right\}$ which represents measurements of a quantity $\left\{v_{i}\right\}$ is given by the sum of the signal variance $\left(\Sigma_{x}^{2}\right)$ and a random variance $\left(\sigma_{x}^{2}\right)$ as

$$
\eta_{x}^{2}=\Sigma_{x}^{2}+\sigma_{x}^{2} .
$$

Likewise, the variance $\left(\eta_{y}^{2}\right)$ of a data set $\left\{y_{i}\right\}$ which represents measurements of the same quantity $\left\{v_{i}\right\}$ is given by

$$
\eta_{y}^{2}=\Sigma_{y}^{2}+\sigma_{y}^{2},
$$

and we can show that

$$
\eta_{x y}=g_{0} \Sigma_{x}^{2} .
$$

where $\eta_{x y}$ is the covariance of $\left\{x_{i}\right\}$ and $\left\{y_{i}\right\}$. We can also show that

$$
\sigma_{x}=\eta_{x}\left(1-\frac{g_{x}}{g_{0}}\right)^{1 / 2},
$$

and

$$
\sigma_{y}=\eta_{y}\left(1-\frac{g_{0}}{g_{y}}\right)^{1 / 2}
$$

where $g_{x}$ and $g_{y}$ are the slopes of least square regression fits to $\left\{x_{i}\right\}$ against $\left\{y_{i}\right\}$ and $\left\{y_{i}\right\}$ against $\left\{x_{i}\right\}$, and $g_{0}$ is the actual slope if all errors are taken into account. More details related to this approach to fitting may be found in Hocking et al. (2001). 
For a time series $a(t)$, the RMS contiguous error $\gamma$ is given by (see, e.g., Holdsworth and Reid 2004b)

$$
\gamma=\sqrt{\sum_{i=1}^{M} \frac{(a(t+1)-a(t))^{2}}{M}}
$$

and we can use this as an estimate of $\eta$. We now apply this approach to our meteor-meteor, and meteor-MF radar wind comparisons.

\section{Davis $33.2 \mathrm{MHz}$ meteor and $55 \mathrm{MHz}$ meteor radar wind comparisons}

Figure 2 shows a scatter plot for the zonal wind component of the wind vector measured by the two Davis meteor radars for 2006 for a height of $88 \mathrm{~km}$. Hourly averages are plotted. Recall that there are only about $22 \%$ of detections in the $55 \mathrm{MHz}$ radar results when compared to the $33.2 \mathrm{MHz}$ radar results, and note the form of the height distributions, with far fewer counts toward 80 and $98 \mathrm{~km}$. The correlation values, contiguous root mean square (RMS), and the number of points are shown in these plots.

The regression of the $55 \mathrm{MHz}$ results on the $33.2 \mathrm{MHz}$ results, and subsequently the regression of $33.2 \mathrm{MHz}$ results on the $55 \mathrm{MHz}$ results, yields two slope estimates for the line of best fit to the data, and these are shown as the red and blue lines, respectively, in the figure. As described above, neither of these lines are correct fits to the data, as the least squares fit analysis assumes that one set of data contains no error, but the correct fit is bounded by these lines. If we assume that both sets of measurements contain equal error and apply a total least square fit to the data, then we can calculate an estimated value for the true slope estimate $g_{0}$, and this is plotted as the green line in the figure. This then allows us to determine the values of the variance in the $33.2 \mathrm{MHz}$ results $\left(\sigma_{x}\right)$ and in the $55 \mathrm{MHz}$ results $\left(\sigma_{y}\right)$. The righthand panel in Fig. 1 shows the relationship between $\sigma_{x}$, $\sigma_{y}$, and $g_{0}$ graphically for the data shown in the left-hand panel. Note that the green dashed lines on the right-hand panel correspond to the total least square fit estimate of $g_{0}$ shown as a solid green line in the left-hand panel. The $\sigma_{x}=\sigma_{y}$ line (which is not plotted to improve clarity) lies halfway between the vertical dash dot black line (which corresponds to $g_{0}=1$ ) and the vertical dashed green line in this plot.

The results of applying this process to the hourly averaged winds for 2006 winds for both wind components for $86 \mathrm{~km}$ are shown in Fig. 2, and Fig. 3 summarizes the results for the entire height range. The panels in Fig. 4 show (from left to right and top to bottom) the correlation coefficients, the ratio of zonal and meridional wind magnitudes measured by the 55 and $33.2 \mathrm{MHz}$ meteor radars, the values of $\sigma_{x}$ and $\sigma_{y}$, the RMS contiguous errors $\gamma_{x}$, and $\gamma_{y}$, the values of $\sigma_{x}$ and $\sigma_{y}$, when $g_{0}=1.0$, the value of $g_{0}$, assuming that $\sigma_{x}=\sigma_{y}$, and the slopes of the standard linear regressions of the $55 \mathrm{MHz}$ result against the 33.2 $\mathrm{MHz}$ radar, and the $33.2 \mathrm{MHz}$ radar against the $55 \mathrm{MHz}$. The results for 2005 and 2007 are very consistent with these results and so are not shown but may be found in McIntosh (2010). Inspection of the first panel in this figure shows a higher correlation in the meridional wind component than the zonal component and shows that the zonal wind component correlations are more consistent over the height range than the meridional component correlations.

We show the mean ratios $(y / x)$ of the wind components for the zonal and meridional components of the $55 \mathrm{MHz}(y)$ to $33.2 \mathrm{MHz}(x)$ systems for completeness and ease of comparison with previous studies. These were calculated for each height bin using an outlier rejection scheme with a 2.5 standard deviation rejection criterion and only where $x \neq 0$. The results show a value consistently less than one. In addition to assuming zero error in one parameter, the mean ratio estimate is more susceptible to the influence of outliers than the regression method for determining $g_{0}$, so additional caution must be taken when using it as a metric.

The RMS contiguous error $\gamma$ is a measure of the error between contiguous time points of the data set. The plots for the RMS contiguous error in Fig. 4 show the results for both zonal and meridional components of both systems. The black and red curves correspond to the 33.2 $\mathrm{MHz}$ meteor system, and the green and blue curves correspond to the $55 \mathrm{MHz}$ system. It is quite clear from the plots that this error is greater for the $55 \mathrm{MHz}$ system than the $33.2 \mathrm{MHz}$ system. This result is reasonable given that the meteor mode operation of the $55 \mathrm{MHz}$ system is interleaved with other non-meteor experiments, increasing the time interval between detections of meteors and subsequent wind samples.

Looking at the plot of the gain factor $g_{0}$, we see that the meridional value is closer to 1.0, suggesting that the assumption $\sigma_{x}=\sigma_{y}$ is not unreasonable, despite the zonal component showing a result not as good. This enables us to estimate the error associated with the random noise components $\delta x_{i}$ and $\delta y_{i}$ for the 33.2 and $55 \mathrm{MHz}$ systems, respectively. The results in Fig. 4 provide a means of characterizing the error associated with the meteor radar winds from each system which we can later apply to the comparisons with the MF PR radar system winds.

The plots of $\sigma_{x}$ and $\sigma_{y}$ when $g_{0}=1$ shown in Fig. 4 use the assumption that there is a $1: 1$ correspondence between the measurements of the signal component $v$ in both data sets with variance $\Sigma_{v}^{2}$. This enables us to 


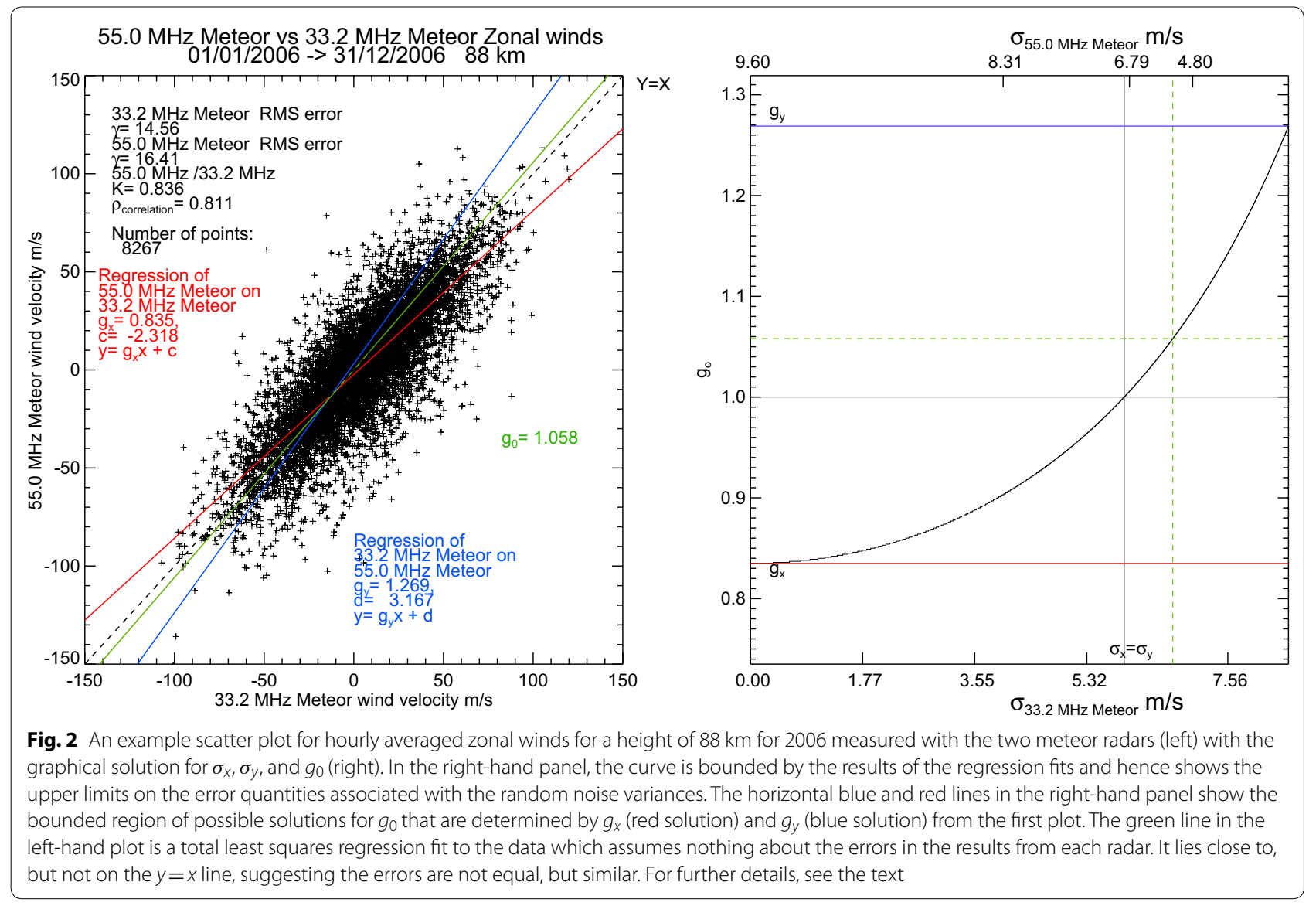

estimate the error associated with the random noise component of each of the data sets. The results are similar to those where the assumption of $\sigma_{x}=\sigma_{y}$ was made. A point to note from this plot is that the error associated with the random noise component of the $55 \mathrm{MHz}$ system $\left(\sigma_{y}\right)$ decreases below that of the $33.2 \mathrm{MHz}$ system between 86 and $90 \mathrm{~km}$ in the meridional direction. Recall that the RMS contiguous error contains both the signal error $\left(\Sigma_{v}^{2}\right)$ and the noise error $\left(\sigma_{v}^{2}\right)$. This shows a gradual increase with height which suggests that the variance $\left(\Sigma_{y}^{2}\right)$ associated with the signal component $(\{v\})$ of the meridional component must increase over this height region in order to maintain the same RMS contiguous error. Inspecting both plots for the RMS contiguous error and the plot for $\sigma_{x}$ and $\sigma_{y}$ when $g_{0}=1$, where both the zonal and meridional errors are comparable in both plots, suggests that the signal variance for the $33.2 \mathrm{MHz}$ measurements $\left(\Sigma_{y}^{2}\right)$ is consistent in both components. However, this is not the case over the entire height range for the variance $\Sigma_{v}^{2}$ for the $55 \mathrm{MHz}$ measurements. These results suggest that there is incomplete sampling of the wind field, that is, $\{v\}$, which increases the variance $\Sigma_{v}^{2}$ of the signal $\{v\}$ as observed.
If we consider the final plot in Fig. 4, two interesting features are present. In the results of the regression of the $55 \mathrm{MHz}$ data on the $33.2 \mathrm{MHz}$ data (black and red curves), there is almost a constant result over the height range, whereas for the results from the regression of $33.2 \mathrm{MHz}$ on $55 \mathrm{MHz}$ there is more of an approach to unity at the peak height before diverging. This suggests that the echo rate at each time-height bin has a significant impact on the quality of the wind estimates produced by the radars. Given that the zonal and meridional components of the $33.2 \mathrm{MHz}$ system show better agreement than the $55 \mathrm{MHz}$ system, this could imply that there is some form of systematic error associated with $55 \mathrm{MHz}$ system. Looking at the overall results for the $55 \mathrm{MHz}$ system, it would suggest that this error would have to be associated with the zonal component of the system, which would further explain more of the observed scatter in the zonal measurements scatter plot.

\section{Davis meteor and MF O-mode wind comparison}

We compared 2 years (2006 and 2007) of meteor and MF winds, but as the results are very similar, we present only the 2006 results here in Figs. 5 and 6. In the interests 


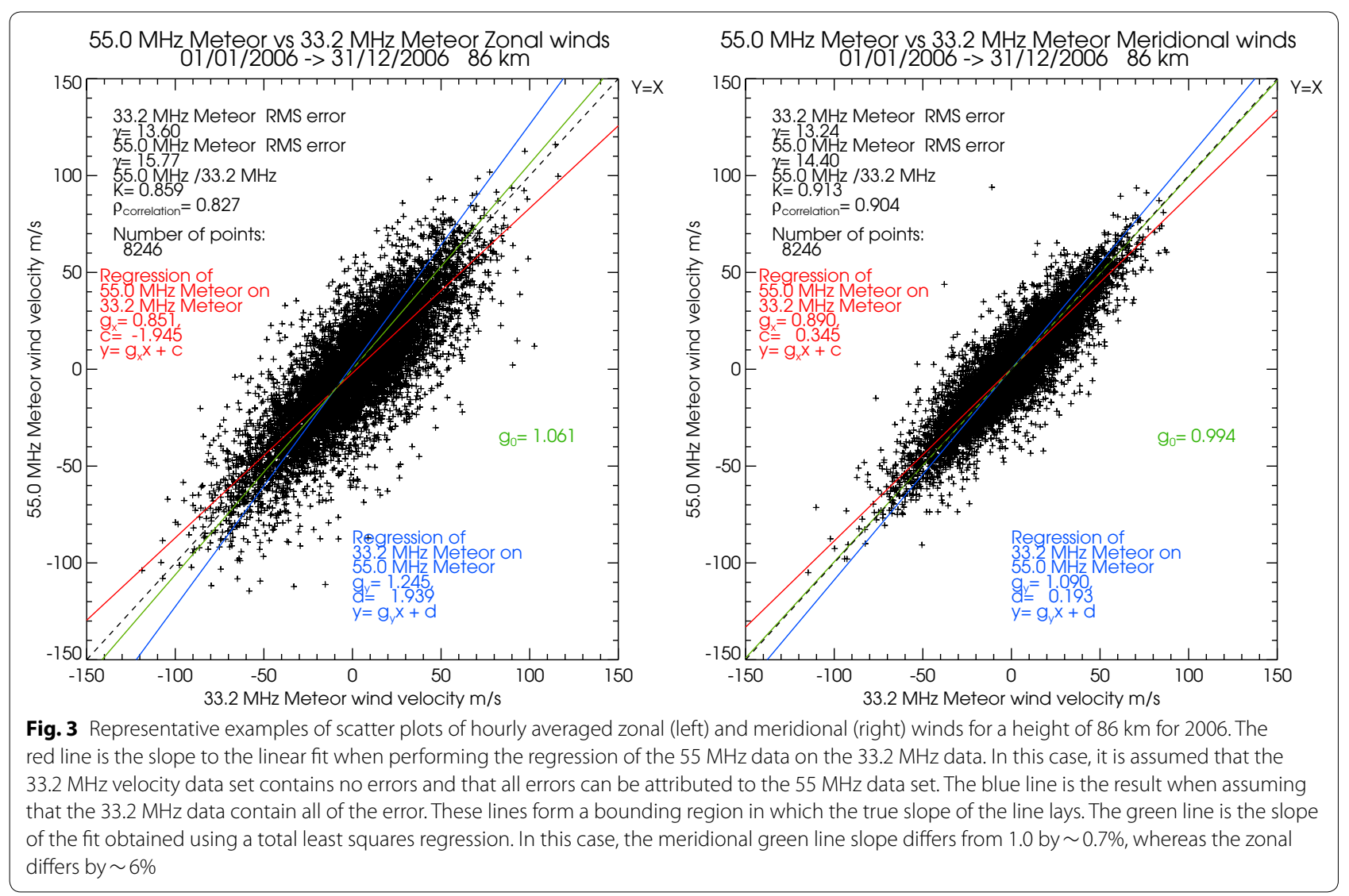

of brevity, we also show only the summary results for $55 \mathrm{MHz}$ meteor and MF O-mode comparisons in Fig. 7. The complete results can be found in McIntosh (2010).

Scatter plots for heights of 80 and $88 \mathrm{~km}$ between the meteor radar winds and the MF O-mode winds are summarized in Fig. 5. These plots show the results of the regression of MF O-mode data on the meteor data (red line) and the meteor data on the MF O-mode data (blue line). The green line is the gain factor $g_{0}$ where the equal error assumption has been made. At this point, it is not clear that this assumption is appropriate, and the result has been included for completeness along with providing an indication of the quality of this assumption in this case.

Inspection of Fig. 6, which summarizes the information from the scatter plots, shows that the meteor MF comparisons show correlations of between 0.7 and 0.8 for 2006 (and 0.7-0.9 for 2007) below $92 \mathrm{~km}$. Unlike the comparison between the two meteor systems, where there was a consistent difference between the zonal and meridional results over the entire height range, there is better agreement between these components, and so in the wind directions, in the comparisons between MF $\mathrm{O}$-mode and meteor data. Both zonal and meridional components show similar correlation values from 86 to $98 \mathrm{~km}$ in 2006 (and 88-98 km in 2007). The mean ratios of the wind components show a gradual decline with increasing height above $82 \mathrm{~km}$ in both 2006 and 2007 for the $33.2 \mathrm{MHz}$ MF O-mode comparison, but less so with the $55 \mathrm{MHz}$ MF O-mode comparison, as shown in Fig. 7.

From the comparison of the two meteor systems above, where the assumption that $\sigma_{x}=\sigma_{y}$ was made, an 'average' value for $\sigma_{x}=\sigma_{y}=\sigma$ was determined over the height range of $80-90 \mathrm{~km}$, where we consider the error to be constant to a first-order approximation. Subsequently, $g_{0}$ was determined from the substitution of the average value of $\sigma$ into the regression equations described above and used in the comparison of the MF O-mode and meteor data. This result is plotted as the purple line in Fig. 5.

The slope of the purple line is close to that of the green line in Fig. 5, which suggests that the assumption of equal error between the meteor and MF O-mode observations may be a way to estimate errors and $g_{0}$ to a first order.

In some previous MF and meteor comparisons (Hocking and Thayaparan 1997, Tsutsumi and Aso 2005), it was observed that underestimates in the MF FCA winds became larger at heights greater than about $90 \mathrm{~km}$. From 
2005 Davis

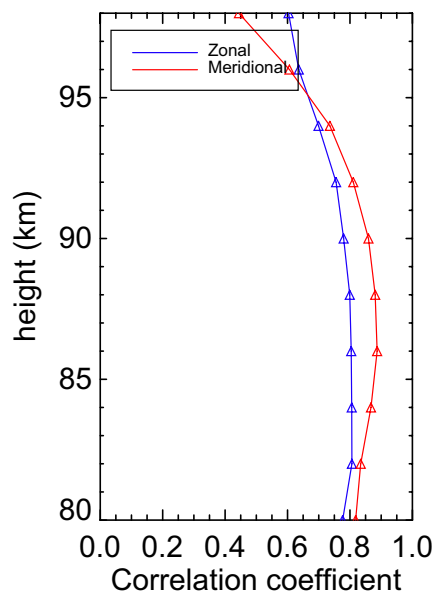

$\mathrm{X}=33.2 \mathrm{MHz}$ Meteor $\mathrm{Y}=55.0 \mathrm{MHz}$ Meteor
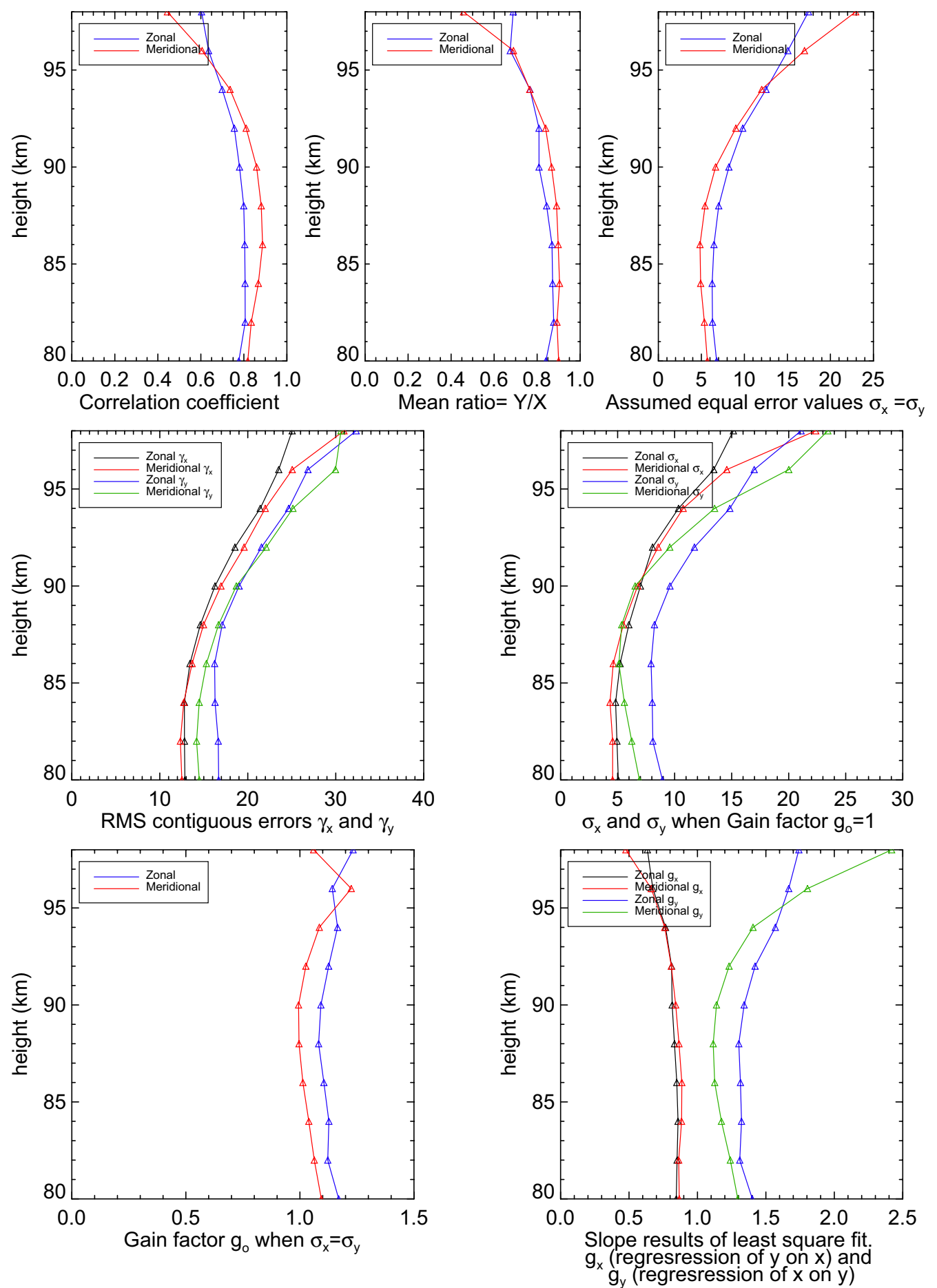

Fig. 4 Summary plot for all heights for 2005 for the parameters shown in Fig. 3. The panels show (from left to right and top to bottom) the correlation coefficients, the ratio of zonal and meridional wind magnitudes measured by the 55 and $33.2 \mathrm{MHz}$ meteor radars, the values of $\sigma_{x}$ and $\sigma_{y}$, the RMS contiguous errors $\gamma_{x}$ and $\gamma_{y}$, the values of $\sigma_{x}$ and $\sigma_{y}$, when $g_{0}=1.0$, the value of $g_{0}$ assuming that $\sigma_{x}=\sigma_{y}$, and the slopes of the standard linear regressions of the $55 \mathrm{MHz}$ results against the $33.2 \mathrm{MHz}$ radar and the $33.2 \mathrm{MHz}$ radar against the $55 \mathrm{MHz}$. In the fourth, fifth, and last panels, red and black indicate the $33.2 \mathrm{MHz}$ radar and green and blue the $55 \mathrm{MHz}$ radar results 

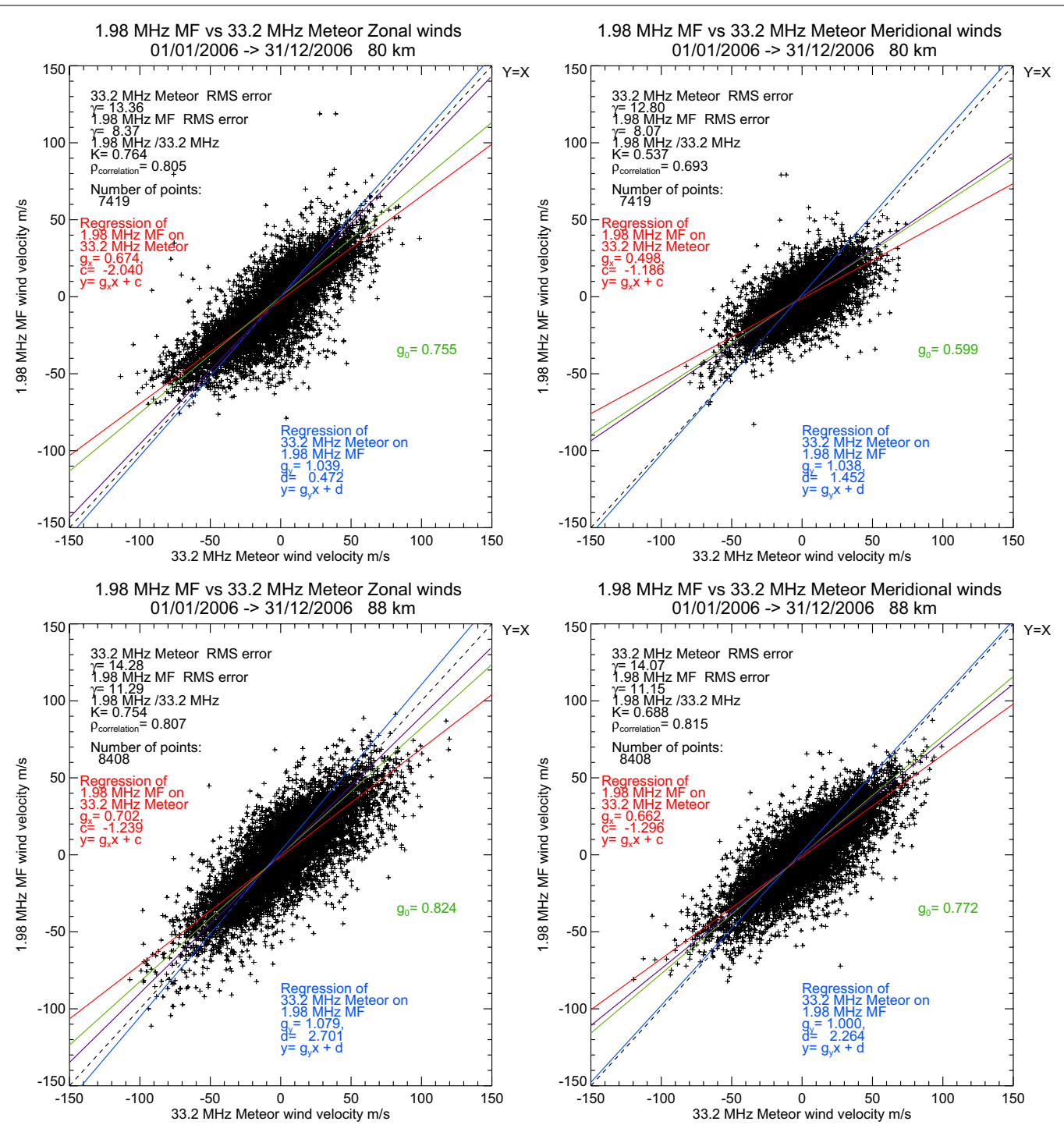

Fig. 5 Davis MF O-mode and 33.2 MHz meteor scatter plot. The purple line represents the slope derived using the error values from the meteormeteor comparison when equal error between both meteor radar measurements was assumed

the results of the mean ratio of the winds and the estimation of $g_{0}$ in the MF O-mode to $33.2 \mathrm{MHz}$ meteor comparison, there is a constant decrease in mean ratio and $g_{0}$ starting at $82 \mathrm{~km}$. In contrast, the results for the $55 \mathrm{MHz}$ and MF O-mode comparison exhibit more consistent values between 82 and $90 \mathrm{~km}$, with a more rapid decrease in these quantities above $90 \mathrm{~km}$. This result is more consistent with observations made by Hocking and Thayaparan (1997) and Tsutsumi and Aso (2005).

The results in Fig. 6 show more consistency between the zonal and meridional components between the two systems. An interesting result can be seen in the plots for $\sigma_{x}$ and $\sigma_{y}$ when $g_{0}=1.0$. In 2006 from $88 \mathrm{~km}$ (and in 2007 from $92 \mathrm{~km}$ ), the error in the meridional component drops to zero. In this case, the scatter plot indicates that the slope relating both measured data values drops well below 1.0, and as such, the result from the regression of $x$ on $y$ is greater than 1.0, which means upon inversion the value for $g_{y}$ is less than 1.0. This implies $g_{0}=1.0$ would not be a valid solution. The values for $g_{y}$ in these cases are typically within $0.3 \%$ of 1.0 , and as such in the interest of maintaining a bounded solution for Eq. (5), we have used $g_{0}=1.0$ as a further bounding solution and set $g_{y}=1.0$ in these cases. This results in $\sigma_{y}=0$ as observed in the plot of $\sigma_{x}$ and $\sigma_{y}$ for $g_{0}=1.0$ in Fig. 6 . It is believed that this arises because of the surd relationship between $\sigma_{x}$, $\sigma_{y}$, and $g_{0}$, and the inability to solve surd relations with $100 \%$ accuracy. This result would also be consistent with 


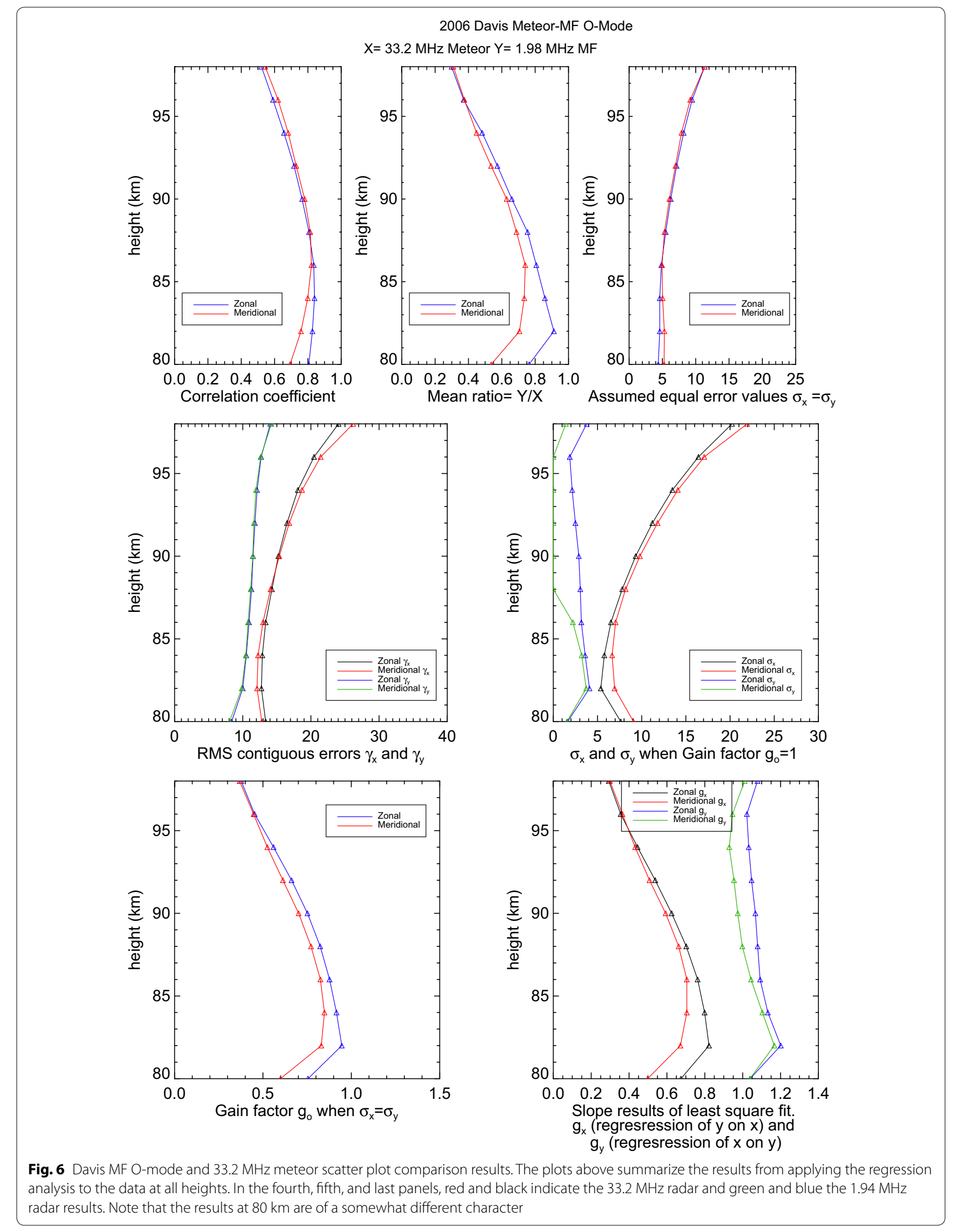




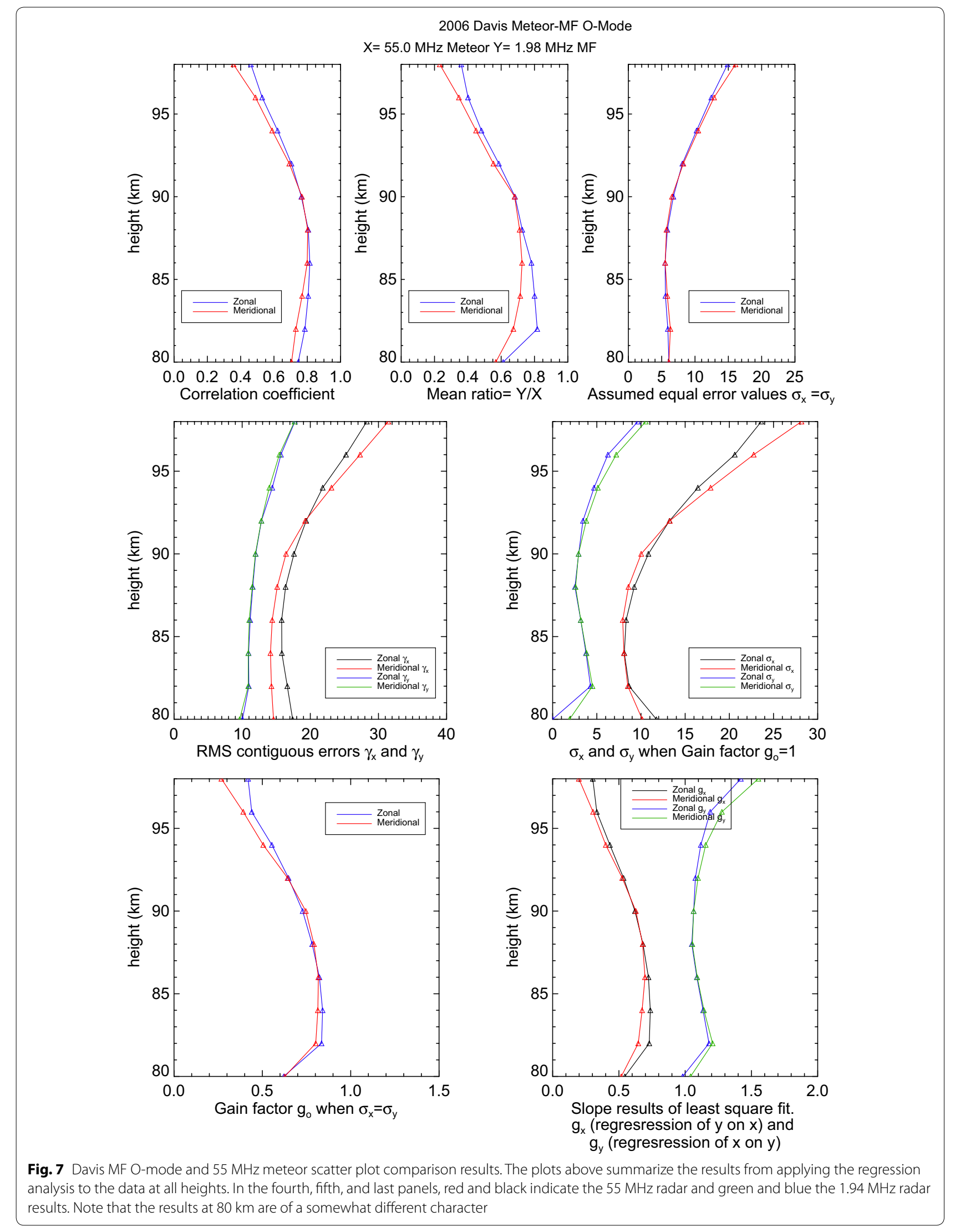


the $33.2 \mathrm{MHz}$ winds being overestimated. Coupled with the known underestimation biases present in the MF SA winds, this would lead to a strong skewing of the scatter that results in an estimate for $g_{y}$ less than 1.0.

An interesting result is evident in Fig. 5, where near the peak height we can make the assumption that both data sets $\{x\}$ and $\{y\}$ contain equal random noise error in both meteor MF O-mode comparisons. This can be seen by the stronger agreement between the green and purple lines in Fig. 5. This agreement is more evident between the $55 \mathrm{MHz}$ meteor and MF O-mode comparison than with the corresponding $33.2 \mathrm{MHz}$ meteor and MF O-mode comparison. This could further suggest a common bias between the $55 \mathrm{MHz}$ and MF O-mode, a result consistent with observations of better agreement between zonal and meridional components of the statistical quantities from the scatter analysis of the $55 \mathrm{MHz}$ meteor and MF O-mode compared with the $33.2 \mathrm{MHz}$ and MF O-mode comparison. Kumar et al. (2007) found in the comparison between a $35.25 \mathrm{MHz}$ meteor radar and MF radar at Thumba $\left(8.5^{\circ} \mathrm{N}, 77^{\circ} \mathrm{E}\right)$, that below $90 \mathrm{~km}$, the MF radar appeared to underestimate the wind by approximately $12 \%$ and approximately $78 \%$ above $90 \mathrm{~km}$. The results for $g_{0}$ in Fig. 6 agree with those found by Kumar et al. (2007). There is a noticeable decrease in the value for $g_{0}$ above $90 \mathrm{~km}$, but it is not as great as that observed in the $55 \mathrm{MHz}$ meteor MF O-mode comparison.

Histograms of the velocity differences between the $55 \mathrm{MHz}$ meteor and MF winds, and $33.2 \mathrm{MHz}$ meteor and MF winds were calculated. The results were found to be Gaussian distributed with similar widths in each component and in each case. The differences between the mean meteor radar and the MF radar winds could be characterized by a shift in the mean, which suggests that a simple height-dependent correction of the mean could validly be applied to correct the MF results in these data. Dolman and Reid (2014) derived a power law correction for the wind magnitude for FCA SA winds in the boundary layer and troposphere. They did not find a height dependence in their results. In preliminary work, Reid and Dolman (2014) compared 1 year of BP $55 \mathrm{MHz}$ meteor radar and MF SA radar winds for 2014 to determine a power law correction for wind magnitude that was also height dependent.

\section{Buckland Park $55 \mathrm{MHz}$ meteor MF wind comparisons}

In order to better understand the representativeness of the Davis results, wind estimates from the BP $55 \mathrm{MHz}$ meteor radar were compared with those obtained using a section of the colocated square kilometer array MF radar operating in SA FCA mode for 2006 and 2007. In 2006, the meteor radar was operated for a four-month period using a single VTX PA module (peak power of about
$18 \mathrm{~kW}, 4 \%$ duty cycle) except during a brief time where an ATRAD STX II (peak power of about $20 \mathrm{~kW}, 10 \%$ duty cycle) transmitter was used. In 2007, the radar was operated for a three-month period by combining all six PA modules from the VTX transmitter (measured peak power of about $83 \mathrm{~kW}$ at a $3 \%$ duty cycle). After 2007, the VTX transmitter was replaced with $40 \mathrm{~kW}, 10 \%$ duty cycle STXII transmitter.

Unlike the Davis $55 \mathrm{MHz}$ meteor system, during these observations the BP $55 \mathrm{MHz}$ meteor system was operated in dedicated meteor mode. The combination of this mode of operation and the higher transmit power level resulted in a higher observed echo rate in each hourheight data bin over the observation period. Average daily values for 2006 were around 7000 with an average peak detection of 1200 near $89 \mathrm{~km}$. In 2007, the average daily count was around 11,000 , with a peak detection of 2000 near $88 \mathrm{~km}$. This should reduce any statistical error in wind estimates associated with low echo rates for the majority of the height distribution. It should also serve to reduce any undersampling of the wind field, which could result in a smaller observed signal variance.

Figure 8 shows only the results for 2007 . We note that the correlation values from the meteor and MF FCA comparison in 2006 are quite similar to the 2007 results, but there is generally a higher correlation in the upper heights in 2007. The RMS contiguous error measurements agree with the Davis meteor MF comparisons but tend to be more constant over the height range, even more so in 2007 when the radar was producing more power. Together with the Davis comparison, this suggests that the echo rate, particularly in the extremities of the height distribution, plays an important role in the statistical reliability of the wind estimates. Both techniques have approximately the same random noise error over the $80-98 \mathrm{~km}$ height range, with a better approximation between 86 and $90 \mathrm{~km}$. The best return height for the MF radar is $86 \mathrm{~km}$ (where the receiver gains are optimized) which is close to the peak height of the meteor distribution. The significance of this is reflected in the correlation and mean ratio plot, where these quantities are maximized, and in the plots for $\sigma_{x}$ and $\sigma_{y}$, where these values are at or close to their minimum values.

These results are in good agreement with those of Cervera and Reid (1995) where it was found that the best agreement between MF and meteor wind velocity estimates was at $86 \mathrm{~km}$. Cervera and Reid $(1995,1996)$ do not present results for the meridional component of the wind. In addition, the meteor radar used by Cervera and Reid $(1995,1996)$ was the original BP narrow beam ST radar which utilized beam steering techniques (see, e.g., Hobbs et al. 2001), as opposed to the all-sky interferometric meteor system used in this comparison. The 

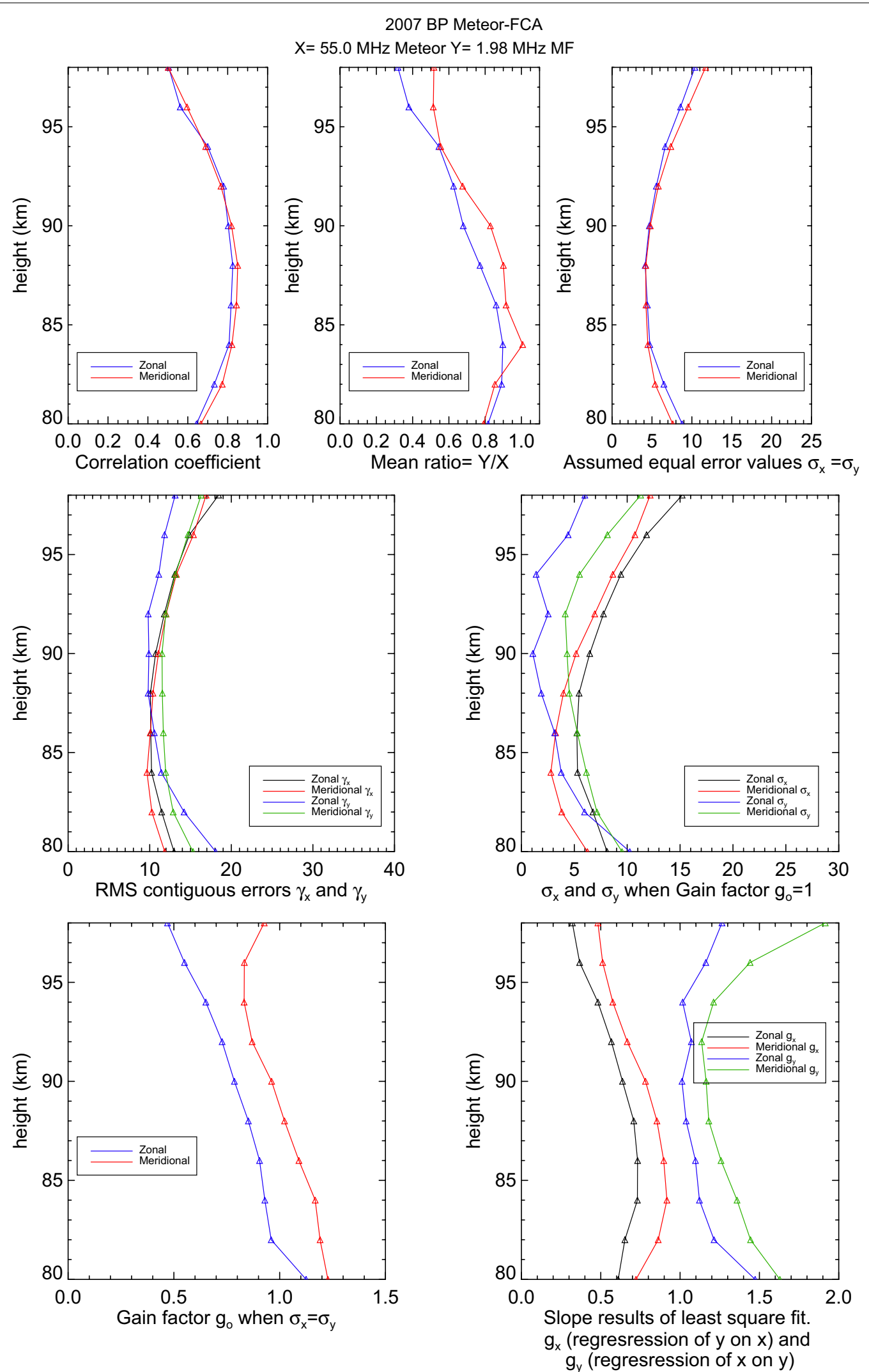

Fig. 8 BP $55 \mathrm{MHz}$ meteor and MF PR FCA wind comparison for 2007. The plots above summarize the results from applying the regression analysis to the data at all heights. In the fourth, fifth, and last panels, red and black indicate the $55 \mathrm{MHz}$ radar and green and blue the $1.98 \mathrm{MHz}$ radar results 
beam was steered off-zenith $60^{\circ}$ in the zonal direction, and interferometric angle of arrival determination was not used.

In the present work for 2006, the values for $g_{0}$ when we assume equal error are close to 1.0, but fall with increasing height. In 2007, these values increase slightly. Given that $g_{0}$ is less than 1.0 for the majority of the height range, particularly in the $88-90 \mathrm{~km}$ height range where the meteor technique produces its strongest results, this indicates that the MF FCA wind estimates are underestimated, as they are for the BP observations of Cervera and Reid $(1995,1996)$, Holdsworth and Reid (2004a, b). Inspection of the plots for the mean ratio and $g_{0}$ for 2006 and 2007 shows that the MF wind estimates are between 10 and $20 \%$ less than the meteor winds at $86 \mathrm{~km}$.

Cervera and Reid (1996) included both slopes and ratios using a total least squares approach and effectively calculated $g_{0}$ assuming equal errors in their work. They found that for 1 year of observations covering 1994, the MF FCA zonal wind to meteor magnitude $\left(g_{0}\right)$ was 0.9 at $80 \mathrm{~km}$ and 0.6 at $98 \mathrm{~km}$. Here, we find that $g_{0}$ for the zonal components in 2006 is 0.9 at $80 \mathrm{~km}$ and 0.5 at $98 \mathrm{~km}$ and in 20071.1 at $80 \mathrm{~km}$ and 0.5 at $98 \mathrm{~km}$. We note that the systems used by Cervera and Reid $(1995,1996)$ are both different from those we have used here, but the consistency of the results is encouraging and suggests a tentative height-dependent calibration factor for BP.

The plot of the regression results in Fig. 8 along with the results from a histogram analysis (not shown) indicates that there is better overall agreement between wind estimates from both radars in the meridional rather than the zonal direction. Similar results can be found in Tsutsumi and Aso (2005). The better agreement found in the meridional component appears to be common with the southern hemisphere comparisons presented in this study. The results of Kumar et al. (2007) do show better agreement in the zonal results as do those of Hall et al. (2005).

The histograms of velocity differences indicate that the mean difference between the two data sets significantly changes between 2006 and 2007. The standard deviation of the distribution of velocity differences, however, appears to remain the same.

\section{Summary and conclusions}

We have made several long-term comparisons of mesospheric wind measurements using different radar systems and different techniques for observing the wind field. A unique comparison of winds measured between two colocated meteor radars along with comparisons between meteor radar wind observations and MF PR SA FCA radar winds has been presented. While the latter comparison has been made previously on several occasions, the colocation of the three instruments at Davis Station provided a pathway for further understanding the differences between winds estimated by both techniques. Access to the three systems permitted a reduction in the number of unknowns in the regression analysis (i.e., $\sigma_{x}, \sigma_{y}$ or $g_{0}$ ) and hence a further clarification and quantification of the statistical relationship between the winds measured using the meteor and MF radar techniques.

The Davis Station comparison of the winds measured by the two collocated meteor radars also provides a measure of the uncertainties associated with the meteor radar technique. It is limited in that the radars differ in their operational frequencies, powers, and observational sequences, but it is still a useful addition to our understanding. The results show very good agreement at the peak heights of the meteor distribution, with errors increasing away from the peak. The influence of using only linear transmit and receive antennas on the detectability of meteors as the distribution rotates during the day remains to be investigated. It may account for the better agreement between the meteor meridional wind components, but this is not certain. This agreement is more evident with the Davis $55 \mathrm{MHz}$ meteor comparisons, possibly due to the interleaved experiment sequence run on that radar and the typically lower echo rates when compared with observations made at $33.2 \mathrm{MHz}$. Although echo rate appears to be a major contributor to the quality of meteor wind estimates over the height range of the meteor distribution, other factors such as sampling sequences, geophysical phenomena, system configuration, and seasonal variations in count rate are believed to play a strong part in the quality of these estimates.

One other limitation of the present work is related to the rotation of the peak detection region of the meteor distribution in the sky during each day. This rotation means that at times (and more so near the edges of the detection height region) the quality of the estimates of the wind velocities will vary semidiurnally. Here, we assume that the error is constant throughout the data set, and this might not be the case. This could be explored by repeating the analysis based on blocks of local time, but we leave this for future work.

The assumption of equal errors for the meteor radars seems reasonable and has allowed us to assign an error to the meteor technique in order to better understand the extent of the underestimation of the wind magnitudes in the MF radar winds. The meteor MF wind comparisons for both Davis Station and BP are consistent with the results of numerous studies and with our introductory points. The Gaussian form of the velocity differences between the techniques suggests that the $g_{0}$ values we have determined using our assumed meteor wind errors 
are suitable for use as calibration values at least when applied to climatologies.

Comparisons between meteor and both MF O-mode and $\mathrm{X}$-mode observations at Davis Station have served to highlight differences in the quality of wind estimates of different polarization modes for these MF radar observations, and we have not included the X-mode analysis here. It is clear from this work that MF radars need to be calibrated, and we have provided linear calibration factors for Davis Station and Buckland Park. The quality of the MF radar winds between 60 and $80 \mathrm{~km}$ remains to be investigated.

\begin{abstract}
Abbreviations
AOA: angle of arrival; BP: Buckland Park; DAE: differential absorption experiment; DPE: differential phase experiment; E-Mode or X-mode: extraordinary mode; FCA: full correlation analysis; IDI: imaging Doppler interferometry; MF: medium frequency; MLT: mesosphere lower thermosphere; O mode: ordinary mode; PA: power amplifier; PMSE: polar mesosphere summer echoes; PR: partial reflection; PRF: pulse repetition frequency; RADAR: radio detection and ranging; RMS: root mean square; SA: spaced antenna; ST: stratosphere troposphere; STXII: solid-state transmitter type 2; VHF: very high frequency; VTX: valve transmitter.
\end{abstract}

\section{Authors' contributions}

IMR wrote this paper based on work conducted by DLM during the latter's $\mathrm{PhD}$ research. IMR and RAV supervised DLM during his PhD candidature. DJM provided the Davis $33.2 \mathrm{MHz}$ data. RAV and DJM contributed to writing this paper. All authors read and approved the final manuscript.

\section{Author details}

${ }^{1}$ ATRAD Pty Ltd, Thebarton, Australia. ${ }^{2}$ University of Adelaide, Adelaide, Australia. ${ }^{3}$ Australian Antarctic Division, Kingston, TAS, Australia.

\section{Acknowledgements}

Funding for this research was provided by the Australian Research Council grants A69943065, DP0450787, DP0878144, and DP1096901, by the Adelaide University ARC Small Grants Scheme, and by ATRAD Pty Ltd. The involvement of IMR was supported by ATRAD Pty Ltd. Operation of the Davis radars has been supported under AAS projects 2529, 2668, and 4025. DLM was supported by an Adelaide University postgraduate scholarship. The loan of the VTX transmitter was facilitated by Ray Morris of the AAD for use at Buckland Park during 2006 and 2007 and is gratefully acknowledged.

\section{Competing interests}

IMR is Executive Director of ATRAD Pty Ltd, which manufactured the radars used in this study.

\section{Ethics approval and consent to participate} Not applicable.

\section{Publisher's Note}

Springer Nature remains neutral with regard to jurisdictional claims in published maps and institutional affiliations.

Received: 22 February 2018 Accepted: 15 May 2018 Published online: 21 May 2018

\section{References}

Briggs BH (1984) The analysis of spaced sensor records by correlation techniques. In: Handbook for MAP, 13, 166-186, international council of scientific unions (SEE N85-17452 08-46), https://ntrs.nasa.gov/archive/ nasa/casi.ntrs.nasa.gov/19850009143.pdf

Cervera MA, Reid IM (1995) Comparison of simultaneous wind measurements using collocated VHF meteor radar and MF spaced antenna radar systems. Radio Sci 30:1245-1261. https://doi.org/10.1029/95RS00644

Cervera MA, Reid IM (1996) A preliminary long term comparison of MF SA and meteor wind determinations with collocated radar systems. In: Seventh workshop on scientific and technical aspects of MST/ST radar, handbook for STEP, SCOSTEP secretariat, University of Illinois, Urbana, USA, pp 158-161

Dolman BK, Reid IM (2014) Bias Correction and overall performance of a VHF spaced antenna boundary layer profiler for operational weather forecasting. J Solar Terr Phys Atmos. https://doi.org/10.1016/j.jastp.2014.02.009i

Hall CM, Aso T, Tsutsumi M, Nozawa S, Manson AH, Meek CE (2005) A comparison of mesosphere and lower thermosphere neutral winds as determined by meteor and medium frequency radar at $70^{\circ} \mathrm{N}$. Radio Sci 40:RS4001. https://doi.org/10.1029/2004RS003102

Hobbs BG, Reid IM, Holdsworth DA (2001) A comparison of tropospheric VHF Doppler beam steering and full correlation analysis measurements of aspect sensitivity. Radio Sci. 36(5):955-964. https://doi.org/10.1029/2000R S002360

Hocking WK (1997) Strengths and limitations of MST radar measurements of middle-atmosphere winds. Ann Geophys 15:1111-1122. https://doi. org/10.1007/s00585-997-1111-1

Hocking WK, Thayaparan T (1997) Simultaneous and colocated observation of winds and tides by MF and meteor radars over London, Canada (43N, 81 ${ }^{\circ}$ W), during 1994-1996. Radio Sci 32(2):833-865. https://doi. org/10.1029/96RS03467

Hocking WK, Thayaparan T, Franke SJ (2001) Method for statistical comparison of geophysical data by multiple instruments which have differing accuracies. Adv Space Res 27:1089-1098. https://doi.org/10.1016/S0273 $-1177(01) 00143-0$

Holdsworth DA, Reid IM (2004a) The Buckland Park MF Radar: final Implementation, observation schemes and velocity comparisons. Ann Geophys 22:3815-3828. https://doi.org/10.5194/angeo-22-3815-2004

Holdsworth DA, Reid IM (2004b) Comparisons of full correlation analysis (FCA) and imaging doppler interferometry (IDI) winds using the Buckland Park MF Radar. Ann Geophys 22:3829-3842. https://doi.org/10.5194/angeo $-22-3829-2004$

Holdsworth DA, Briggs BH, Reid IM, Vincent RA (1996) Intercomparisons of various spaced antenna analyses. In: Seventh workshop on scientific and technical aspects of MST/ST radar, handbook for STEP, SCOSTEP Secretariat, University of Illinois, Urbana, USA, pp 430-433

Holdsworth DA, Vincent RA, Reid IM (2001) Mesospheric turbulent velocity estimation using the Buckland Park MF radar. Ann Geophys 19(8):10071017. https://doi.org/10.5194/angeo-19-1007-2001

Holdsworth DA, Vuthaluru R, Reid IM, Vincent RA (2002) Differential absorption measurements of mesospheric and lower thermospheric electron densities using the Buckland Park MF radar. J Atmos Solar Terr Phys 64:20292042. https://doi.org/10.1016/S1364-6826(02)00232-8

Holdsworth DA, Reid IM, Cervera MA (2004a) The Buckland Park all-sky interferometric meteor radar-description and first results. Radio Sci 39:RS5009. https://doi.org/10.1029/2003RS003014

Holdsworth DA, Tsutsumi M, Reid IM, Nakamura T, Tsuda T (2004b) Interferometric meteor radar phase calibration using meteor echoes. Radio Sci. 39:RS5012. https://doi.org/10.1029/2003RS003026

Holdsworth DA, Morris RJ, Murphy DJ, Reid IM, Burns GB, French WJR (2006) Antarctic mesospheric temperature estimation using the Davis VHF radar. J Geophys Res 111:D05108. https://doi.org/10.1029/2005JD006589

Holdsworth DA, Murphy DJ, Reid IM, Morris RJ (2008) Antarctic meteor observations using the Davis MST and meteor radars. Adv Space Res 42:143-154. https://doi.org/10.1016/j.asr.2007.02.037

Jones GOL, Berkey FT, Fish CS, Hocking WK, Taylor MJ (2003) Validation of imaging Doppler interferometer winds using meteor radar. Geophys Res Lett 30:1743. https://doi.org/10.1029/2003GL017645

Kumar KK, Rankumar G, Shelbi ST (2007) Initial results from SKiYMET meteor radar at Thumba $\left(8.5^{\circ} \mathrm{N}, 77^{\circ} \mathrm{E}\right): 1$. Comparison of wind measurements with MF spaced antenna radar system. Radio Sci 42:RS6008. https://doi. org/10.1029/2006RS003551

Manson AH, Meek CE, Brekke A, Moen J (1992) Mesosphere and lower thermosphere $(80-120 \mathrm{~km})$ winds and tides from near Troms $\left(70^{\circ} \mathrm{N}, 19^{\circ} \mathrm{E}\right)$ : 
comparisons between radars (MF, EISCAT, VHF) and rockets. J Atmos Ter Phys 54(7-8):927-950. https://doi.org/10.1016/0021-9169(92)90059-T

McIntosh DL (2010) Comparisons of VHF meteor radar observations in the middle atmosphere with multiple independent remote sensing techniques, Ph.D. Dissertation, University of Adelaide, http://hdl.handl e.net/2440/60068

McKinley DWR (1961) Meteor science and engineering. McGraw-Hill, New York Reid IM (1988) MF Doppler and spaced antenna measurements of upper middle atmosphere winds. J Atmos Terr Phys 50:117-134. https://doi. org/10.1016/0021-9169(88)90049-9

Reid IM (1990) Radar observations of stratified layers in the mesosphere and lower thermosphere (50-100 km). Adv Space Res 10:7-19. https://doi. org/10.1016/0273-1177(90)90002-H

Reid IM (2015) MF and HF Spaced Antenna radar techniques for investigating the dynamics and structure of the 50 to $110 \mathrm{~km}$ height region: a review. Prog Earth Planet Sci 2:33. https://doi.org/10.1186/s40645-015-0060-7

Reid IM, Vincent RA (1987) Measurements of mesospheric gravity wave momentum fluxes and mean flow accelerations at Adelaide, Australia. J Atmos Terr Phys 49:443-460. https://doi.org/10.1016/00219169(87)90039-0

Reid IM, Dolman BK (2014) The MF radar technique revisited. In: Paper 04-114 presented at the 14th international workshop on technical and scientific aspects of MST radar, INPE, May 25-31, 2014, Sao Jose dos Campos/SP, Brazil
Reid IM, Vandepeer BGW, Dillon SD, Fuller BG (1995) The new adelaide MF Doppler radar. Radio Sci 30:1177-1189. https://doi.org/10.1029/95RS0 0731

Reid IM, Holdsworth DA, Morris RJ, Murphy DM, Vincent RA (2006) Meteor observations using the Davis MST radar. J Geophys Res. https://doi. org/10.1029/2005JA011443

Tsutsumi M, Aso T (2005) MF radar observations of meteors and meteorderived winds at Syowa $\left(69^{\circ} \mathrm{S}, 39^{\circ} \mathrm{E}\right)$, Antarctica: a comparison with simultaneous spaced antenna winds. J Geophys Res 110:D24111. https:// doi.org/10.1029/2005JD005849

Wilhelm S, Stober G, Chau JL (2017) A comparison of 11-year mesospheric and lower thermospheric winds determined by meteor and MF radar at $69^{\circ} \mathrm{N}$. Ann Geophys 35:893-906. https://doi.org/10.5194/angeo-35-893-2017

Younger JP, Reid IM (2017) Interferometer angle-of-arrival determination using precalculated phases. Radio Sci 52:1058-1066. https://doi. org/10.1002/2017RS006284

Younger JP, Reid IM, Vincent RA, Holdsworth DA (2008) Modeling and observing the effect of aerosols on meteor radar measurements of the atmosphere. Geophys Res Lett 35:L15812. https://doi.org/10.1029/2008G L033763

Younger JP, Reid IM, Vincent RA (2013) Mutual coupling of antennas in a meteor radar interferometer. Radio Sci 48:118-121. https://doi. org/10.1002/rds.20026

\section{Submit your manuscript to a SpringerOpen ${ }^{\odot}$ journal and benefit from:}

- Convenient online submission

- Rigorous peer review

- Open access: articles freely available online

- High visibility within the field

- Retaining the copyright to your article

Submit your next manuscript at $\boldsymbol{\nabla}$ springeropen.com 